\title{
Sarcoma hepático embrionario indiferenciado: reporte de caso y revisión de la literatura
}

\author{
Vilma M. Pérez-Rodríguez ${ }^{\star}$, Teodoro Muñiz-Ronquillo², Marco A. Ponce-Camacho ${ }^{3}$ y \\ Claudia Y. Rodríguez-Garza ${ }^{4}$ \\ ${ }^{1}$ Departamento de Pediatría; ${ }^{2}$ Centro Universitario Contra el Cáncer; ${ }^{3}$ Servicio de Anatomía Patológica y Citopatología; ${ }^{4}$ Centro Universitario de \\ Imagen Diagnóstica. Hospital Universitario Dr. José Eleuterio González, Monterrey, Nuevo León, México
}

\begin{abstract}
Resumen
Introducción: El sarcoma hepático embrionario indiferenciado representa el 9-13\% de los tumores hepáticos malignos en la edad pediátrica y es la tercera neoplasia maligna primaria de hígado en la infancia. Sin embargo, son pocos los casos reportados en la literatura. Se puede manifestar con fiebre, pérdida de peso, dolor y sensación de tumor abdominal. Los estudios de imagen, además de los estudios anatomopatológico e inmunohistoquímico, son las herramientas adecuadas para el diagnóstico. Caso clínico: Se presenta el caso de una paciente de 6 años de edad con diagnóstico de sarcoma hepático embrionario indiferenciado mediante cirugía y posterior resultado de la biopsia. Conclusiones: Al revisar la literatura se encontró que este tipo de neoplasia maligna no es frecuente en la infancia. Sin embargo, es importante considerar este tipo de tumor como causa en aquellos casos de hepatomegalia en la edad pediátrica.
\end{abstract}

Palabras clave: Sarcoma hepático embrionario indiferenciado. Neoplasia maligna. Inmunohistoquímica.

\section{Undifferentiated embryonal liver sarcoma: case report and review of the literature}

\begin{abstract}
Background: Undifferentiated embryonal sarcoma of the liver accounts for 9-13\% of malignant tumors in the pediatric age group, and is the third primary malignant neoplasm of the liver in children. However, few cases are reported in the literature. It may manifest with fever, weight loss, pain, and abdominal tumor sensation. In addition to pathology and immunohistochemistry, imaging studies are the appropriate tools for diagnosis. Case report: We present the case of a 6-year-old female patient diagnosed with undifferentiated embryonal sarcoma of the liver by surgery and subsequent biopsy results. Conclusions: When reviewing the literature, we found that this type of malignant neoplasm is not frequent in children. However, it is important to consider this type of tumor as a cause in those cases of hepatomegaly in the pediatric age.
\end{abstract}

Keywords: Undifferentiated embryonal sarcoma of the liver. Malignant neoplasm. Immunohistochemistry.

Correspondencia:

*Vilma M. Pérez-Rodríguez

E-mail: vilma_marcela@ hotmail.com

(http://creativecommons.org/licenses/by-nc-nd/4.0/)
Fecha de recepción: 25-01-2021

Fecha de aceptación: 03-05-2021

DOI: 10.24875/BMHIM.21000011
Disponible en internet: 16-12-2021

Bol Med Hosp Infant Mex. 2021;78(6):647-651

www.bmhim.com 


\section{Introducción}

Las neoplasias primarias de hígado representan aproximadamente el $0.5-2 \%$ de los tumores en la edad pediátrica ${ }^{1}$. En 1978, Stocker e Ishak ${ }^{2}$ describieron el sarcoma hepático embrionario indiferenciado como una condición clínico-patológica que se subdivide en rabdomiosarcoma biliar, angiosarcoma, rabdoide e indiferenciado (Tabla 1) (3,4. $^{3}$.

Esta enfermedad se manifiesta principalmente en niños entre los 5 y 12 años de edad, con una distribución similar en ambos sexos. Después del hepatoblastoma y del carcinoma hepatocelular, el sarcoma hepático ocupa el tercer lugar de frecuencia, con un $9-13 \%$. Se puede presentar con las siguientes manifestaciones clínicas: disnea, pérdida de peso, mal estado general, dolor epigástrico y tumor hepático palpable de grandes dimensiones, predominantemente en el lóbulo hepático derecho ${ }^{5}$. Las pruebas de función hepática tienden a resultar normales o ligeramente elevadas, y pocas veces los marcadores tumorales, como la alfa-fetoproteína y la fracción beta de la gonadotropina coriónica humana, se encuentran incrementados ${ }^{6}$.

El ultrasonido, la tomografía computada (TC) y la resonancia magnética son relativamente inespecíficas. En general muestran una lesión sólida, heterogénea, que puede presentar áreas quísticas (necrosis) o hemorragia, y algunas veces la lesión se observa avascular. El diagnóstico definitivo se confirma mediante histopatología con tinción de hematoxilina-eosina y análisis inmunohistoquímico, que es positivo para CD56, CD68, vimentina y desmina?.

Histopatológicamente se observan elementos mesenquimatosos malignos sin ninguna evidencia de diferenciación específica. Dicha variabilidad histológica dificulta el diagnóstico y origina diferentes nombres: mesenquimoma maligno, rabdomiosarcoma del hígado, fibromiosarcoma, sarcoma indiferenciado o sarcoma embrionario. La relación entre el hamartoma y el sarcoma, así como con el hepatoblastoma, es un factor predisponente ${ }^{8,9}$. Este tipo de tumores son típicamente grandes en el momento del diagnóstico y difíciles de resecar ${ }^{10}$.

El comportamiento clínico de este tumor es agresivo, pero potencialmente tratable con terapia multimodal, incluyendo quimioterapia, cirugía, radioterapia y, en casos específicos, trasplante de hígado ${ }^{11}$. Los regímenes de quimioterapia incluyen doxorubicina, ifosfamida, ciclofosfamida, vincristina y etopósido ${ }^{12}$.

Los resultados de los estudios prospectivos de grupos europeos publicados en la última década
Tabla 1. Diagnóstico diferencial de neoplasias malignas hepáticas según sus marcadores inmunohistoquímicos

\begin{tabular}{|l|l|}
\hline Neoplasia hepática maligna & $\begin{array}{l}\text { Marcadores } \\
\text { inmunohistoquímicos }\end{array}$ \\
\hline Hepatoblastoma & Ki-67 \\
\hline $\begin{array}{l}\text { Carcinoma hepatocelular } \\
\text { HepPar-1, glipican-3, } \\
\text { arginasa, HSP70, CD34 }\end{array}$ \\
\hline $\begin{array}{l}\text { Sarcoma } \\
\text { Rabdomiosarcoma biliar } \\
\text { Rabiosarcoma } \\
\text { Indiferenciado }\end{array}$ & $\begin{array}{l}\text { CK7, CK19, } \\
\text { CD31, CD34, citoqueratina } \\
\text { CD31, CD 34 } \\
\text { Vimentina, desmina, CD56, } \\
\text { CD68 }\end{array}$ \\
\hline $\begin{array}{l}\text { Metástasis } \\
\text { Tumor de Wilms }\end{array}$ & $\begin{array}{l}\text { CK8, CK18, CK20 } \\
\text { mioglobina, desmina }\end{array}$ \\
\hline Neuroblastoma & Enolasa \\
\hline Colorrectal & CK20 \\
\hline Tumor carcinoide & Cromogranina, sinaptofisina \\
\hline $\begin{array}{l}\text { Hemangioendotelioma } \\
\text { kaposiforme }\end{array}$ & CD31, CD34 \\
\hline $\begin{array}{l}\text { Linfohistiocitosis } \\
\text { Lemofagocítica }\end{array}$ & CD68 \\
\hline $\begin{array}{l}\text { Leucemia megacarioblástica } \\
\text { CDise células de }\end{array}$ & S-100, CD1-a \\
\hline
\end{tabular}

confirman la baja frecuencia de este diagnóstico dentro de los tumores hepáticos malignos en niños.

Si bien la supervivencia ha mejorado, la biología y el comportamiento agresivo del tumor condicionan aún una alta mortalidad.

En el año 2002 se reportaron los resultados de 17 pacientes con diagnóstico de sarcoma hepático indiferenciado tratados entre 1979 y 1995 por el grupo colaborativo italiano y alemán de sarcomas en tejidos blandos. Diez de los 17 pacientes (58\%) se encontraban vivos con un seguimiento de entre 2.4 y 20 años. De los pacientes en que se documentó alguna recaída, uno de ellos aún vivía después de 4 años de la recurrencia y había respondido adecuadamente.

\section{Caso clínico}

Se presenta el caso de una paciente de 6 años, previamente sana, con antecedente familiar de cáncer cervicouterino por rama materna. Inició su padecimiento actual 2 semanas antes de su ingreso, al 


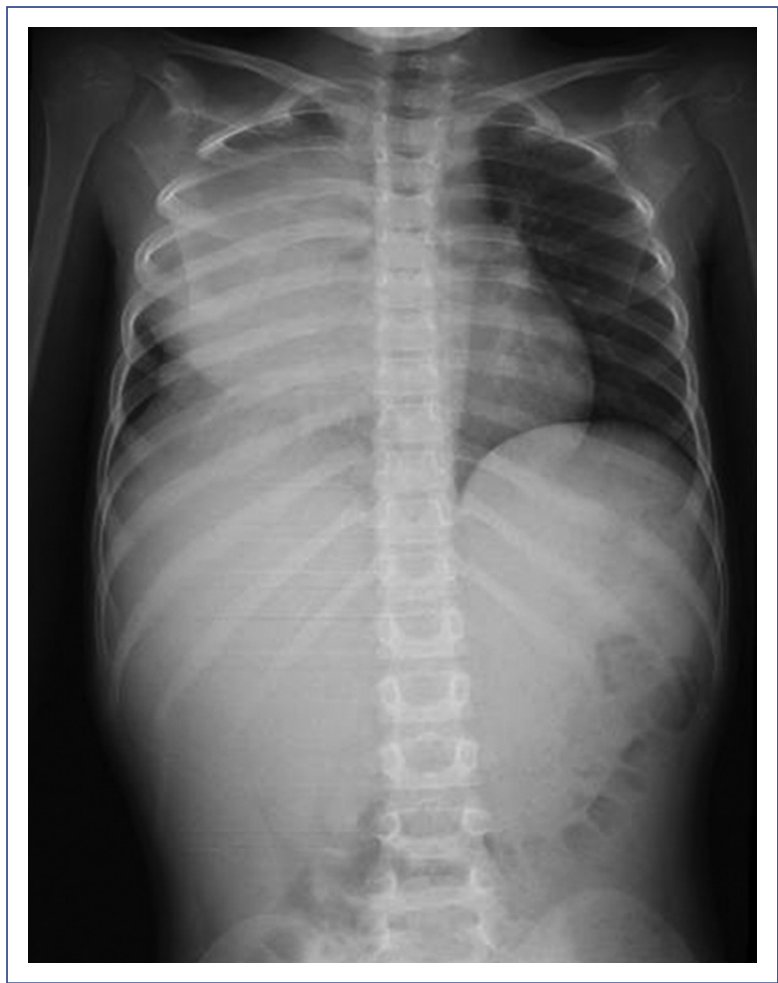

Figura 1. Radiografía de tórax con radioopacidad del hemitórax derecho, signo de silueta y atelectasia adyacente.

presentar fiebre intermitente de predominio nocturno. Al acudir a su médico particular, este solicitó una radiografía de tórax que evidenció una radioopacidad que ocupaba casi la totalidad del hemitórax derecho, con signo de silueta respecto a las estructuras cardiomediastínicas y atelectasia del pulmón adyacente (Figura 1), por lo que fue remitida a un hospital de tercer nivel.

A su ingreso en el hospital, la exploración física reveló hepatomegalia $>5 \mathrm{~cm}$, no dolorosa, fija a planos profundos, sugerente de tumor hepático. Los estudios de laboratorio reportaron anemia microcítica hipocrómica, pruebas de función hepática normales, y fosfatasa alcalina y lactato deshidrogenasa elevadas. A continuación se realizó una TC toracoabdominal contrastada en la que se evidenció hepatomegalia condicionada por una tumoración de $14 \times 12 \times 7.8 \mathrm{~cm}$ que afectaba los segmentos VIII, V, IVa y el lóbulo caudado, predominantemente hipodensa, de aspecto sólido, asociada con áreas de necrosis y hemorragia. Con un borde lobulado y circunscrito, y presencia de calcificaciones periféricas, la lesión se extendía hacia la zona supradiafragmática con ocupación del hemitórax

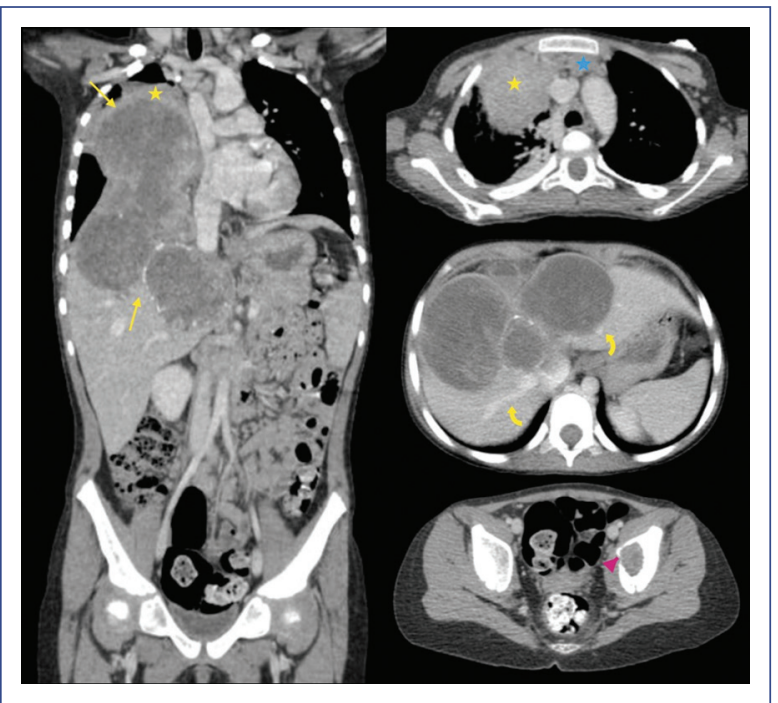

Figura 2. Tomografía computada toracoabdominal contrastada. Cortes sagital y axial que evidencian una tumoración hepática con extensión supradiafragmática derecha $(\Rightarrow)$, atelectasia del lóbulo medio derecho $(\xi)$ íntimo contacto con las venas suprahepáticas derecha e izquierda con involucro de la media ( $\mathscr{0})$, adenopatías mediastinales ( y) lesión lítica en el hueso ilíaco izquierdo $(\checkmark)$.

derecho, condicionando el desplazamiento del parénquima pulmonar y atelectasia del lóbulo medio derecho. Además, la lesión mostraba extensión anterior, posicionándose adyacente a la pared abdominal y en íntimo contacto con las venas suprahepáticas derecha e izquierda; también involucraba la vena suprahepática media, comprimiendo la vena cava inferior y la aurícula izquierda. Asimismo, se identificaron múltiples adenopatías mediastinales y una lesión lítica con componente de tejidos blandos en el hueso ilíaco izquierdo (Figura 2).

Por sospecha de neoplasia hepática maligna, se solicitó su valoración por los servicios de oncología y cirugía pediátrica. Debido a la fiebre persistente, se sospechó un absceso hepático, por lo que se realizó un ultrasonido abdominal en el que se reportaron múltiples lesiones hepáticas. Se solicitó una biopsia, que se realizó por radiointervencionismo guiado por ultrasonido con búsqueda intencionada de Entamoeba. Se reportó necrosis masiva de aspecto neoplásico, además de la prueba serológica negativa. Se evaluaron los marcadores tumorales indicados por parte de oncología pediátrica: alfa-fetoproteína de $1.74 \mathrm{ng} / \mathrm{mL}$ y fracción beta de la gonadotropina coriónica humana $<0.10 \mathrm{mUl} / \mathrm{mL}$, ambos dentro de los valores normales. 


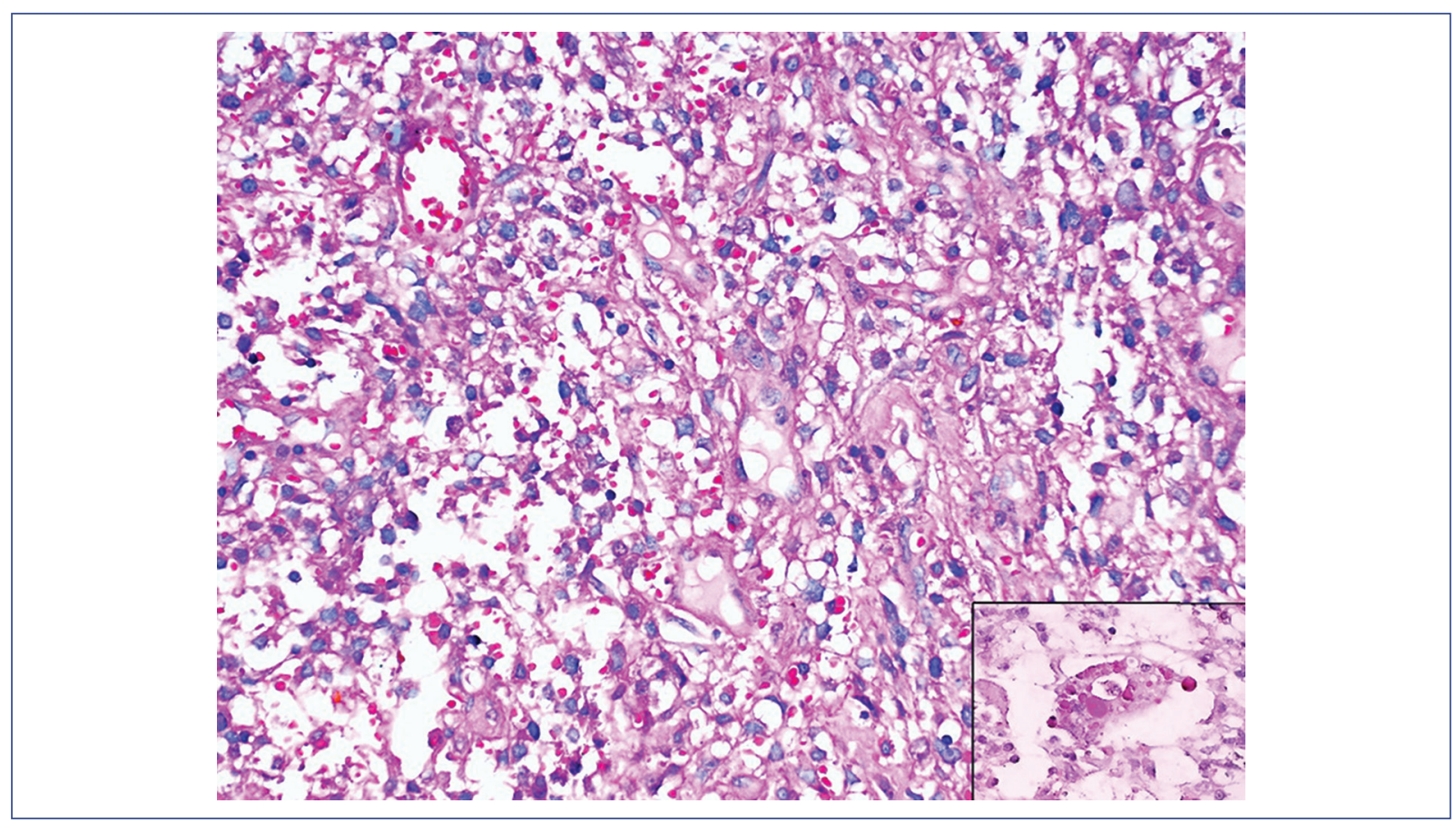

Figura 3. Neoplasia mesenquimatosa primitiva compuesta por células redondas a ovales indiferenciadas. Se identificaron glóbulos hialinos ocasionales (recuadro) (tinción con hematoxilina y eosina 40x).

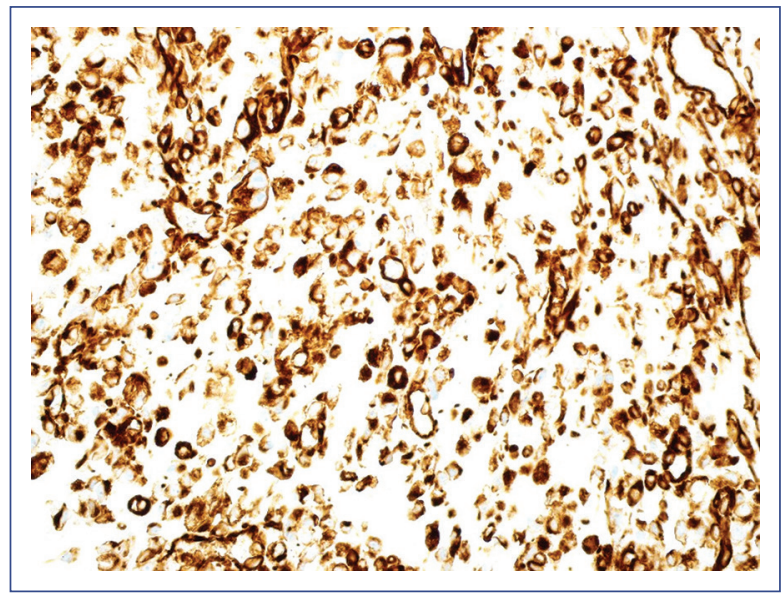

Figura 4. Células neoplásicas fuertemente positivas para vimentina $(40 \times)$.

Con respecto a la evolución de la paciente, presentó compromiso neurológico a expensas de crisis convulsivas tónico-clónicas, aparentemente relacionadas con alteraciones electrolíticas. Se solicitó estudio de imagen que descartó la infiltración al sistema nervioso central. Se realizó una nueva biopsia a cielo abierto, en la que se observó un tumor con aspecto de racimo de uvas en el epiplón. El reporte de patología informó un diagnóstico de sarcoma hepático embrionario indiferenciado con inmunohistoquímica positiva para vimentina y negativa para desmina, CD56 y CD58 (Figuras 3 y 4). Posteriormente se inició tratamiento de quimioterapia con vincristina, ifosfamida y etopósido. La paciente cumplió nueve ciclos y presentó buena tolerancia y buena respuesta clínica, aunque pobre respuesta radiológica.

Se realizó una nueva TC toracoabdominal contrastada, en la que se observó la persistencia del tumor hepático (con respuesta a la quimioterapia), pero aún con involucro vascular de la vena suprahepática media y el efecto de masa de la vena cava inferior, además de la aurícula izquierda. La lesión lítica en la región ilíaca izquierda se encontró de menor tamaño.

A pesar de la pobre respuesta radiológica, la paciente se sometió a la resección del tumor primario y su extensión al tórax. Después de múltiples complicaciones transoperatorias, se logró la resección total de la tumoración. Sin embargo, la evolución fue desfavorable por complicaciones asociadas a un evento de choque hipovolémico hemorrágico, por lo que la paciente falleció 24 horas después del evento quirúrgico. 


\section{Discusión}

El sarcoma indiferenciado hepático es un tumor raro, predominantemente de la edad pediátrica (aunque también puede presentarse en adultos), sin predominio por ningún sexo. En este reporte se describe el caso de una paciente de 6 años que inició con un cuadro febril de larga duración sin foco aparente. Al ser sometida a estudios de imagen se evidenció una masa que comprometía el hemitórax derecho, motivo por el cual fue referida a un hospital de segundo nivel. Se realizó una TC torácica y abdominal en la que se encontró una lesión sugestiva de hepatoblastoma que se correlacionaba con la presencia de una tumoración abdominal durante la exploración física, por lo que fue referida a un hospital de tercer nivel para su abordaje integral. En dicha institución se realizó el abordaje multidisciplinario hasta concluir con un diagnóstico histopatológico de sarcoma hepático indiferenciado, el cual correspondía, según el reporte patológico, con lo descrito en la literatura. Se encontró un tumor de aspecto heterogéneo, solitario y bien circunscrito, con predominio en el lóbulo hepático derecho. Sus características sugerían un origen mesenquimal indiferenciado. En el análisis inmunohistoquímico destacó la presencia del marcador vimentina, lo cual se describe en la literatura. El tratamiento de elección fue combinado, quimioterapia y cirugía, aunque con un pronóstico desfavorable. En esta paciente se descartó la posibilidad de un trasplante ya que, como se ha descrito en la literatura, el tratamiento ideal de estos tumores es la resección ${ }^{13}$.

El sarcoma hepático es una neoplasia agresiva y poco frecuente en la infancia, con síntomas inespecíficos, diagnosticado mediante estudios de imagen y estudio histopatológico de una muestra obtenida por biopsia. El abordaje y el tratamiento deben ser multidisciplinarios, involucrando desde médicos de primer contacto hasta equipos de alta especialidad.

Cabe destacar que el abordaje de los tumores abdominales de probable origen hepático debe ser realizado de acuerdo con algoritmos establecidos en la literatura universal que permitan realizar el diagnóstico de manera oportuna y, por ende, el inicio temprano de un tratamiento. Este tipo de reportes de un tumor hepático de baja frecuencia en la infancia obliga al pediatra de atención primaria a considerar esta patología como una más de las causas de cuadros clínicos sugerentes de neoplasia hepática.

\section{Responsabilidades éticas}

Protección de personas y animales. Los autores declaran que para esta investigación no se han realizado experimentos en seres humanos ni en animales.

Confidencialidad de los datos. Los autores declaran que han seguido los protocolos de su centro de trabajo sobre la publicación de datos de pacientes.

Derecho a la privacidad y consentimiento informado. Los autores han obtenido el consentimiento informado de los pacientes y sujetos referidos en el artículo. Este documento obra en poder del autor de correspondencia.

\section{Conflicto de intereses}

Los autores declaran no tener ningun conflicto de intereses.

\section{Financiamiento}

Ninguno.

\section{Bibliografía}

1. Bisogno G, Pilz T, Perilongo G, Ferrari A, Harms D, Ninfo V, et al. Undifferentiated sarcoma of the liver in childhood: a curable disease. Cancer. 2002;94:252-7.

2. Stocker JT, Ishak KG. Undifferentiated (embryonal) sarcoma of the liver: report of 31 cases. Cancer. 1978;42:336-48

3. Pérez GRM, Herrera MH, León BB, Ortiz HC. Sarcoma indiferenciado embrionario del hígado. Estudio clínico-patológico e inmunohistoquímico de ocho casos con énfasis en el diagnóstico diferencial con tumores intraabdominales en niños y adultos jóvenes. Patol Rev Latinoam. 2011;49:25-32.

4. Meyers LR, Trobaugh-Lotrario AD, Malogolowkin MH, Katzenstein HM, López-Terrada DH, Finegold MJ. Pediatric liver tumors. En: Pizzo AP, Poplack GD, editores. Principles and practice of pediatric oncology. Philadelphia: Wolters Kluwer; 2016. p. 726-52.

5. Vásquez-Gutiérrez E, Calderón-Elvir CA, Ruano-Aguilar JM, Gutiérrez-Ureña JA, Duarte-Valencia JC, Leal-Leal CA. Sarcoma embrionario indiferenciado hepático en niños. Presentación de dos casos clínicos. Rev Oncol. 2002;4:501-5.

6. von Schweinitz D. Management of liver tumor in chilhood. Semin Pediatr Surg. 2006;15:17-24.

7. Quintero-Rodríguez K, Villegas-González V, Pérez-Hidalgo JM, Pérez-Alvarado MC, Forero-Melo J. Sarcoma embrionario indiferenciado en dos pacientes pediátricos. Rev CES Med. 2018;32:301-9.

8. Romero-Cubero D, Corrales LA, Zúñiga P, Rodríguez C. Hepatoblastoma y sarcoma embrionario: revisión y reporte de dos casos. Acta Pediátrica Costarricense. 2004;18:18-23.

9. Urban CE, Mache CJ, Schwinger W, Pakisch B, Ranner G, Riccabona M, et al. Undifferentiated (embryonal) sarcoma of the liver in childhood. Cancer. 1993;72:2511-6.

10. Weinberg AG, Finegold MJ. Primary hepatic tumors of childhood. Human Pathol. 1983;14:512-37.

11. Putra J, Ornvold K. Undifferentiated embryonal sarcoma of the liver: a concise review. Arch Pathol Lab Med. 2015;139:269-73.

12. Techavichit P, Masand PM, Himes RW, Abbas R, Goss JA, Vasudevan SA, et al. Undifferentiated embryonal sarcoma of the liver (UESL): a single-center experience and review of the literature. J Pediatr Hematol Oncol. 2016;38:261-8.

13. Robles R, Marín C, Ramírez P, Sánchez-Bueno F, Parrila P. Indicaciones controvertidas de trasplante hepático: tumores primarios distintos del hepatocarcinoma y metástasis hepática. Med Clin Monogr (Barc). 2007;8:8-15. 